\title{
NOTE ON HOROSPHERES*
}

BY JAMES PIERPONT

In a paper $\dagger$ before the Mathematical Association of America (Sept. 1923) and in another before the International Mathematical Congress at Toronto (August, 1924) I have developed a treatment of a part of non-euclidean geometry which has certain advantages, so it seems to me, over the projective methods of Klein. This method rests on the metric of Riemann and on the introduction of certain variables in terms of which the equations of the straight line and plane are linear.

In the first paper mentioned I showed how easy it is to arrive at Clifford's parallels in elliptic space. These lie on surfaces called Clifford surfaces; their curvature is zero and hence their geometry for restricted regions is euclidean.

In hyperbolic space there are also surfaces of zero curvature, the horospheres of Lobatschevsky and Bolyai. I now wish to show how they may be obtained by the preceding method.

Let $x, y, z$ be ordinary rectangular coördinates. Let $R$ be a positive constant. We set

$$
\begin{gathered}
r^{2}=x^{2}+y^{2}+z^{2}, \lambda=4 R^{2}-r^{2}, \mu=4 R^{2}+r^{2}, \\
d \sigma^{2}=d x^{2}+d y^{2}+d z^{2} .
\end{gathered}
$$

The metric of $H$-space $\ddagger$ as defined by Riemann is given by the equation

$$
d s=\frac{4 R^{2} d \sigma}{\lambda} .
$$

* Presented to the Society, May 1, 1926.

† American Mathematical Monthly, vol. 30, p. 425, and vol. 31, p. 26. The Proceedings of the Toronto Congress have not yet appeared.

$\ddagger$ For $H$-, read hyperbolic; for $e$-, read euclidean. 
Here $R$ plays the role of the space constant and $-1 / R^{2}$ the curvature of this space. Since $d s<0$ for $e$-points without the $e$-sphere $\lambda=0$, we restrict ourselves in $H$-geometry to points within it. The distance from any point within $\lambda$ to a point on it is infinite. It is the boundary of $H$-space. Straight lines or $H$-straights are defined by

$$
\delta \int d s=0 .
$$

They turn out to be $e$-circles cutting the $\lambda$-sphere orthogonally.

In many problems it is convenient to introduce the variables

$$
z_{1}=\frac{4 R^{2} x}{\lambda}, z_{2}=\frac{4 R^{2} y}{\lambda}, z_{3}=\frac{4 R^{2} z}{\lambda}, z_{4}=R \mu / \lambda .
$$

In problems of the plane we may set $z=0$ in $r, \lambda, \mu$ in (1) and use

$$
z_{1}=\frac{4 R^{2} x}{\lambda}, z_{2}=\frac{4 R^{2} y}{\lambda}, z_{3}=R \mu / \lambda .
$$

The $\lambda$ sphere is then replaced by the $\lambda$-circle, $x^{2}+y^{2}-4 R^{2}=0$. With this introduction, let us consider $H$-circles, in the $x y$ plane. Their equation is in $z$-coordinates

$$
a_{1} z_{1}+a_{2} z_{2}-a_{3} z_{3}=k, \text { a constant, }
$$

the center having the $z$-coordinates $a_{1}, a_{2}, a_{3}$. In $x y$ coordinates (5) becomes

(6) $x^{2}+y^{2}+\frac{4 R^{2}}{k-a_{3} R}\left(a_{1} x+a_{2} y\right)-\frac{4 R^{2}\left(k+a_{3} R\right)}{k-a_{3} R}=0$,

which is an $e$-circle.

We ask what happens when the center $a$ converges to a point on the $\lambda$-circle. To answer this question we consider the family of $e$-circles

$$
C_{\alpha}=x^{2}+y^{2}-2 \alpha x-4 R(R-\alpha)=0 .
$$


whose centers lie on the $x$ axis and which touch the $\lambda$-circle at the point $A$; also the $e$-circles

$$
L_{\beta}=x^{2}+y^{2}-4 R x-2 \beta y+4 R^{2}=0 .
$$

These cut the $\lambda$-circle orthogonally and are therefore $H$ straights. They also pass through the point $A$ and cut the $C_{\alpha}$ orthogonally.

We show that the $C_{\alpha}$ may be regarded as $H$-circles whose center is $A$ and whose radii are the $L_{\beta}$. In fact let $\mathrm{L}_{\beta}$ cut $C_{\alpha}$ and $C_{\alpha_{1}}$ in $P, P_{1}$; we show the $H$-length of the segment $P P_{1}$ on the radius $L_{\beta}$ is independent of $\beta$.

For if $L_{\beta}$ cuts $C_{\alpha}$ in the point $P$ whose coordinates are $x, y$ we find that

where

$$
\xi=x-2 R=\frac{2 \beta^{2} c}{\gamma^{2}}, y=\frac{2 \beta c^{2}}{\gamma^{2}},
$$

$$
c=\alpha-2 R, \quad \gamma^{2}=\beta^{2}+c^{2} .
$$

If $L_{\beta}$ cuts the adjacent circle $C_{\alpha+d \alpha}$ in $P^{\prime}$, we find the length of the $\operatorname{arc} P P^{\prime}$ is

$$
d s=\frac{2 R^{2} d \alpha}{\alpha(2 R-\alpha)} ;
$$

hence

$$
\overline{P P}_{1}=\int d s
$$

which is independent of the particular radius $L_{\beta}$.

We may also show that the $H$-circles (5) or (6) converge to the circles $C_{\alpha}$. In fact take $a$ on the $x$ axis for simplicity; then $a_{2}=0$. We may now let $a_{1}, a_{3}, k \rightarrow \infty$ such that

$$
\lim _{k \rightarrow \infty} \frac{a_{1}}{k}=\lim _{k \rightarrow \infty} \frac{a_{3}}{k}=\frac{1}{R} \cdot \frac{\alpha}{\alpha-2 R} .
$$

In this case (6) goes over into a $C_{\alpha}$ circle. These $C_{\alpha}$ circles are horocircles. For simplicity we took their $H$-center $A$ on the $x$ axis; obviously $A$ may be any point on the 
$\lambda$-circle. Any $e$-circle lying within $\lambda=0$ and tangent to it, is a horocircle in $H$-geometry.

To get a horosphere we merely revolve one of the horocircles about the diameter through $A$; i.e., an $e$-sphere lying within the $\lambda$-sphere or $x^{2}+y^{2}+z^{2}-4 R^{2}=0$, and tangent to it is a horosphere. We wish to show that these surfaces have 0 -curvature or that the metric on them has the form

$$
d s^{2}=\frac{4 R^{4} c^{2}}{\alpha}\left(d u^{2}+u^{2} d \varphi^{2}\right)
$$

To this end let us revolve a $C_{\alpha}$ about the $x$ axis getting a horosphere $S_{\alpha}$. The intersection $P$ of $C_{\alpha}$ and some $L_{\beta}$, describes an $e$-circle which we may regard as a parallel of latitude $\beta$ whose pole is $A$ and we may use this as one coordinate. As second coordinate on $S_{\alpha}$ we may use the meridian circles $\varphi$ cut out of $S_{\alpha}$ by $e$-planes through the $x$ axis and making the angle $\varphi$ with the $x z$ plane. If now we set $\rho^{2}=y^{2}+z^{2}$, we have, using (9), (10),

$$
\rho=\frac{2 \beta c^{2}}{\gamma^{2}}, y=\rho \sin \varphi, z=\rho \cos \varphi .
$$

Now $d s$ is given by (2). We find

$$
\begin{gathered}
\beta d \beta=\gamma d \gamma, \quad d x=d \xi, \quad d y^{2}+d z^{2}=d p^{2}+\rho^{2} d \varphi^{2}, \\
d \rho=\frac{2 c^{2}}{\gamma^{4}}\left(c^{2}-\beta^{2}\right) d \beta, d \xi=\frac{4 \beta c^{3}}{\gamma^{4}} d \beta, d \sigma^{2}=\frac{4 c^{4}}{\gamma^{4}}\left(d \beta^{2}+\beta^{2} d \varphi^{2}\right) .
\end{gathered}
$$

Hence

$$
d s^{2}=\frac{4 R^{4} c^{2}}{\alpha^{2}}\left(\frac{d \beta^{2}}{\beta^{4}}+\frac{d \varphi^{2}}{\beta^{2}}\right) .
$$

Set now $\mu=1 / \beta$; then $d s^{2}$ reduces to (13); q.e.d.

YALE UNIVERSITY 